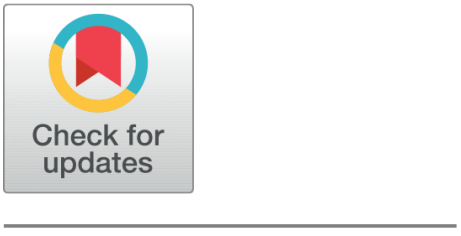

OPEN ACCESS

Received: 22.05.2021

Accepted: 06.07.2021

Published: 30.07 .2021

Citation: Singh BP, Dixit S (2021) Estimation of Risk of Child Death and Average Children Ever Born. Indian Journal of Science and Technology 14(26): 2180-2188. https ://doi.org/10.17485/IJST/v14i26.889

* Corresponding author.

brijesh@bhu.ac.in

Funding: None

Competing Interests: None

Copyright: () 2021 Singh \& Dixit. This is an open access article distributed under the terms of the Creative Commons Attribution License, which permits unrestricted use, distribution, and reproduction in any medium, provided the original author and source are credited.

Published By Indian Society for Education and Environment (iSee)

ISSN

Print: 0974-6846

Electronic: 0974-5645

\section{Estimation of Risk of Child Death and Average Children Ever Born}

\author{
Brijesh P Singh ${ }^{1 *}$, Shweta Dixit ${ }^{2}$ \\ 1 Department of Statistics, Institute of Science, Banaras Hindu University, Varanasi, 221005, \\ India \\ 2 Clinical Development Services Agency (CDSA), NCR Biotech Science Cluster, 3rd Floor, THSTI \\ Building, Faridabad, 121001, India
}

\section{Abstract}

Objectives: To understand the pattern of child death (death before their fifth birthday), in this study an attempt has been made to develop a probability model. Methods: Mortality especially child death is one of the important and interesting areas for population scientists. In this paper, an attempt has been made a probability model to study the pattern of child deaths and also try to estimate the average number of children ever born. The parameters involved in the model under consideration have been estimated with the method of moments. Findings: Some real data sets from NFHS are used for checking the suitability of the proposed model. The performance of proposed model is outstanding for the considered set of data. The risk of child death in Uttar Pradesh is estimated as 63 per thousand and the estimated number of children ever born is about 7 in the reproductive life of a female. In the urban area, the risk of child death and average children ever born is estimated lower than the estimate of rural area. We observed risk of child death and the average number of children ever born is decreasing over time. Novelty: The proposed model is competent to estimate the risk of child death and the average children ever born to a female through the distribution of number of child death.

Keywords: Child Death; Average Children Ever Born; Probability Model; Marginal Probability; Method of Moment

\section{Introduction}

Childhood mortality is one of the important indicators of a nation's general medical and public health conditions, and consequently, the country's level of socio-economic development. Its decline is therefore not only desirable but also indicative of an improvement in general living standards. The infant mortality rate, probability of dying before one year of age expressed per 1000 live-births and under-five mortality rate, probability of dying between birth and age 5 expressed per 1000 live-births have been considered as measures of children's well-being for many years. In India, 2.1 million children die before their fifth birthday (child death). Half of these children die even before they are 28 days old, accounting for one-fourth of global infant deaths. Among the 9.7 million child deaths worldwide annually, one-third occur in India. While around 4 million children die within the first 28 days of life across the world every year, 
India records around one million of these cases. Apart from this infant mortality, child mortality rates have also declined. However, despite this decline, more than 1 child in 18 children still die within the first year of life, and 1 in 13 die before reaching age five. These rates also vary substantially across various states of India. The picture of mortality is highest in the most populous state in India i.e. Uttar Pradesh, which has about 1 in 8 children die before reaching their fifth birthday. If one can identify the responsible biological and socio-demographic factors for child death, the health and intervention program can be reformulated and implemented to reduce the intensity of child death. The Infant and child death is known as a good and sensitive indicator of the development of a nation and the impact of government intervention programs and policies. Child death has a positive impact on fertility ${ }^{(1,2)}$, and reduction in child death or enhancement in the probability of survival of children is one of the known reasons for reduction infertility. That implies a reduction in infant and child morality which is likely the most important aim of the millennium development goals, as children are the most important assets of a nation. Studies on early-age mortality in the last five decades are mostly confined to infant death, but, it has been realized that child death also needs to be examined in addition to infant mortality.

A number of parametric models for the study of the age patterns of mortality have been developed over the year. In order to get this pattern through modeling, the first parametric model for the duration of mortality is developed by ${ }^{(3)}$. The first-ever attempt to study infant mortality through parametric modeling is proposed by ${ }^{(4)}$ who used a hyperbolic function for the study of infant mortality. Another attempt to represent mortality across the entire age range was the eight parameter non-linear model in, "Age pattern of mortality" ${ }^{(5)}$. Later, ${ }^{(6)}$ proposed a logarithmic approximation and the Weibull function is recommended by ${ }^{(7)}$. After that several attempts have been made to study age at infant mortality through mathematical models. In these circumstances, a number of attempts have been made to study the age pattern of the mortality mathematical model ${ }^{(8-11)}$. Modified Polya-Aeppli and Poisson-Gamma distribution for child death is discussed by ${ }^{(12)}$. A modified truncated Geometric distribution was developed for the number of child death ${ }^{(13)}$. Beta-Binomial model and inflated Binomial distribution are proposed for the number of child death for fixed parity i.e. fixed number of children ever born ${ }^{(14,15)}$. Further ${ }^{(16)}$ developed a model by mixing Poisson distribution with the Beta-Binomial distribution to study the variation of child death in society. Poisson-Lindley distribution is proposed for the study of a number of child deaths and compare with Poisson-Exponential and Poisson-Gamma distribution ${ }^{(17)}$. Recently a mixture probability distribution is developed for the study of the pattern of child death ${ }^{(18)}$. In the present study, we have proposed a probability model for child death which gives a clear picture of its pattern. The beauty of this model is that we can estimate the risk of child death as the above mentioned previous models but this model also provides the estimate of average children ever born to the females, which is the added benefit of the proposed model. This study proposes a mathematical model to explain the pattern of child death for all females in the most populous state of India i.e. Uttar Pradesh along with other major states of the different parts of India.

\section{Proposed Model}

Since probability models provide concise and clear representations of extensive data sets in a better way in recent years increased attention has been paid to the proposition and derivation of probability models for the distribution of infant mortality. Let $x$ denote the number of child death to a female and the distribution of a number of child death to females of given parity $\mathrm{k}$ and risk of child death $\mathrm{p}$ follows a binomial distribution.

$$
P[x \mid k, p]=\left(\begin{array}{c}
k \\
x
\end{array}\right) p^{x} q^{k-x} ; 0 \leq p \leq 1, k>0, x=0,1,2, \ldots, k
$$

As we discussed in the literature, that the number of parity (number of children ever born to a female) $k$ itself a random variable and following a displaced Poisson distribution with probability mass function as;

$$
P[K=k]=\frac{e^{-\lambda} \lambda^{k-1}}{(k-1) !} ; k=1,2, \ldots, \infty
$$

Therefore, the joint density of $\mathrm{k}$ and $\mathrm{x}$ for the given $\mathrm{p}$ is given by,

$$
P[K=k \cap r \mid k, p]=P[r \mid k, p] \times P[K=k]==\frac{e^{-\lambda} \lambda^{k}}{(k) !}\left(\frac{k}{\lambda}\right)\left(\begin{array}{c}
k \\
r
\end{array}\right) p^{r} q^{k-r}
$$

Hence, the marginal distribution of $\mathrm{x}$;

$$
P[X=x \mid k]=\sum_{k=x}^{\infty} \frac{e^{-\lambda} \lambda^{k}}{(k) !}\left(\begin{array}{c}
k \\
x
\end{array}\right) p^{x} q^{k-x}\left(\frac{k}{\lambda}\right)
$$

https://www.indjst.org/ 
On simplifying this marginal distribution we get;

$$
P[X=x \mid k]=\frac{e^{-\lambda p}(\lambda p)^{x}}{(x) !}\left(\frac{x}{\lambda}+1-p\right)
$$

Further, in this model, we assume that the probability of death of a child varies from female to female but remains constant at each birth for a given female. We assume that the probability of death of child ' $\mathrm{p}$ ' follows a beta distribution with parameter a and $\mathrm{b}$ which is given by;

$$
f[p]=\frac{1}{\beta(a, b)} p^{a-1}(1-p)^{b-1} ; a, b>0 ; 0 \leq p \leq 1
$$

The joint density of $\mathrm{x}$ is given as;

$$
P[x \cap p]=\frac{e^{-\lambda p}(\lambda p)^{x}}{(x) !}\left(\frac{x}{\lambda}+1-p\right) p^{a-1}(1-p)^{b-1} \frac{1}{\beta(a, b)}
$$

After simplifying this reduces to;

$p[X=x \mid p]=\frac{e^{-\lambda p}(\lambda p)^{x}}{(x) !}\left(\frac{x}{\lambda}\right)\left(\frac{p^{a-1}(1-p)^{b-1}}{\beta(a, b)}\right)+\frac{e^{-\lambda p}(\lambda p)^{x}}{(x) !}\left(\frac{p^{a-1}(1-p)^{b-1}}{\beta(a, b)}\right)-\frac{e^{-\lambda p}(\lambda p)^{x}}{(x) !}\left(\frac{p^{a}(1-p)^{b-1}}{\beta(a, b)}\right)$

Hence, the marginal distribution of $\mathrm{x}$ is given by;

$$
\begin{aligned}
& p[X=x]=\frac{\lambda^{x-1}}{\beta(a, b)(x-1) !} \int_{0}^{1} e^{-\lambda p} p^{a-1}(1-p)^{b-1} d p \\
& +\frac{\lambda^{x}}{\beta(a, b)(x) !} \int_{0}^{1} e^{-\lambda p} p^{a+x-1}(1-p)^{b-1} d p-\frac{\lambda^{x}}{\beta(a, b)(x) !} \int_{0}^{1} e^{-\lambda p} p^{a+x}(1-p)^{b-1} d p
\end{aligned}
$$

It can be verified easily that

$$
\sum_{x=0}^{\infty} P[X=x]=1
$$

\section{Estimation Procedure}

In this study method of the moment has been used as an estimation procedure to estimate the unknown parameters $\lambda$, a, and $\mathrm{b}$ of the model considered for a number of child deaths to females of all parity. The method of moments generally provides an estimate which is consistent but not as efficient as the method of maximum likelihood. But the method of moments is often used because it leads to very simple computations than the maximum likelihood method. Also, the complexities in mathematical derivation involved in the maximum likelihood method. The first three moments of the probability model considered here are as follows

$$
\begin{gathered}
E(x)=\frac{a}{(a+b)}(\lambda+1) \\
E\left(x^{2}\right)=\frac{a(a+1)}{(a+b+1)(a+b)}(\lambda+2) \lambda+\frac{a}{(a+b)}(\lambda+1) \\
E\left(x^{3}\right)=\frac{a(a+1)(a+2)}{(a+b+2)(a+b+1)(a+b)}\left(\lambda^{3}+3 \lambda^{2}\right) \\
+\frac{a(a+1)}{(a+b+1)(a+b)} 3\left(\lambda^{2}+2 \lambda\right)+\frac{a}{(a+b)}(\lambda+1)
\end{gathered}
$$


Let $\mu_{1}^{\prime}, \mu_{2}^{\prime}$ and $\mu_{3}^{\prime}$ denotes the three raw moments about zero for the data, thus replacing $E(x), E\left(x^{2}\right)$ and $E\left(x^{3}\right)$ by $\mu_{1}^{\prime}, \mu_{2}^{\prime}$ and $\mu_{3}^{\prime}$ respectively in the above equations. Hence, we can obtain three equation with three unknown parameters $\lambda, a$ and $b$, as given below

$$
\begin{gathered}
\mu_{1}^{\prime}=\frac{a}{(a+b)}(\lambda+1) \\
\mu_{2}^{\prime}=\frac{a(a+1)}{(a+b+1)(a+b)}(\lambda+2) \lambda+\frac{a}{(a+b)}(\lambda+1) \\
\mu_{3}^{\prime}=\frac{a(a+1)(a+2)}{(a+b+2)(a+b+1)(a+b)}\left(\lambda^{3}+3 \lambda^{2}\right) \\
+\frac{a(a+1)}{(a+b+1)(a+b)} 3\left(\lambda^{2}+2 \lambda\right)+\frac{a}{(a+b)}(\lambda+1)
\end{gathered}
$$

From equation (12) we have,

$$
(a+b)=\frac{a}{\mu_{1}^{\prime}}(\lambda+1)
$$

This implies that

$$
b=\frac{a}{\mu_{1}^{\prime}}(\lambda+1)-a
$$

Using equation (12) and (13) we have;

$$
\begin{gathered}
\mu_{2}^{\prime}=\frac{a(a+1)}{(a+b+1)(a+b)}(\lambda+2) \lambda+\mu_{1}^{\prime} \\
\mu_{2}^{\prime}-\mu_{1}^{\prime}=\frac{a(a+1)}{(a+b+1)(a+b)}(\lambda+2) \lambda=\frac{\mu_{1}^{\prime}(a+1)}{(a+b+1)(\lambda+1)}(\lambda+2) \lambda \\
\frac{\mu_{2}^{\prime}-\mu_{1}^{\prime}}{\mu_{1}^{\prime}}=\frac{(a+1)}{(a+b+1)(\lambda+1)}(\lambda+2) \lambda
\end{gathered}
$$

putting the values of $b$ from (16);

$$
\begin{aligned}
& \frac{\mu_{2}^{\prime}-\mu_{1}^{\prime}}{\mu_{1}^{\prime}}=\frac{(a+1)}{\left[a+\frac{a+a \lambda-a \mu_{1}^{\prime}}{\mu_{1}^{\prime}}+1\right](\lambda+1)}(\lambda+2) \lambda \\
& \left.\Rightarrow \frac{\mu_{2}^{\prime}-\mu_{1}^{\prime}}{\mu_{1}^{2}}=\frac{(a+1)}{\left(a+a \lambda+\mu_{1}^{\prime}\right)(\lambda+1)}(\lambda+2) \lambda=\delta \text { (say }\right)
\end{aligned}
$$

solving the above equation, we get the value of 'a,

$$
\Rightarrow a=\frac{\mu_{1} \delta(\lambda+1)-\lambda(\lambda+2)}{\lambda(\lambda+2)-\delta\left(\lambda^{2}+2 \lambda+1\right)}
$$

Now from equation (14) \& (15), we have;

$$
\mu_{3}^{\prime}=\frac{a(a+1)(a+2)}{(a+b+2)(a+b+1)(a+b)}\left(\lambda^{3}+3 \lambda^{2}\right)+3\left(\mu_{2}^{\prime}-\mu_{1}^{\prime}\right)+\mu_{1}^{\prime}
$$




$$
\begin{gathered}
\mu_{3}^{\prime}-3\left(\mu_{2}^{\prime}-\mu_{1}^{\prime}\right)-\mu_{1}^{\prime}=\frac{a(a+1)(a+2)}{(a+b+2)(a+b+1)(a+b)}\left(\lambda^{3}+3 \lambda^{2}\right) \\
\frac{\mu_{3}^{\prime}-3\left(\mu_{2}^{\prime}-\mu_{1}^{\prime}\right)-\mu_{1}^{\prime}}{\mu_{1}^{\prime}}=\frac{(a+1)(a+2)}{(a+b+2)(a+b+1)} \frac{\left(\lambda^{3}+3 \lambda^{2}\right)}{(1+\lambda)}
\end{gathered}
$$

Using equation (15);

$$
\begin{aligned}
& \frac{\mu_{3}^{\prime}-3\left(\mu_{2}^{\prime}-\mu_{1}^{\prime}\right)-\mu_{1}^{\prime}}{\mu_{1}^{\prime}}=\frac{(a+1)(a+2)}{\left(\frac{a(1+\lambda)}{\mu_{1}^{\prime}}+2\right)\left(\frac{a(1+\lambda)}{\mu_{1}^{\prime}}+1\right)} \frac{\left(\lambda^{3}+3 \lambda^{2}\right)}{(1+\lambda)} \\
& \frac{\mu_{3}^{\prime}-3\left(\mu_{2}^{\prime}-\mu_{1}^{\prime}\right)-\mu_{1}^{\prime}}{\mu_{1}^{3}}=\frac{(a+1)(a+2)}{\left(a+a \lambda+2 \mu_{1}^{\prime}\right)\left(a+a \lambda+\mu_{1}^{\prime}\right)} \frac{\left(\lambda^{3}+3 \lambda^{2}\right)}{(1+\lambda)}=\eta \text { (say) }
\end{aligned}
$$

Simplifying equation (21), we get;

$$
\Rightarrow(a+1)(a+2)\left(\lambda^{3}+3 \lambda^{2}\right)=\eta\left(a+a \lambda+2 \mu_{1}^{\prime}\right)\left(a+a \lambda+\mu_{1}^{\prime}\right)(1+\lambda)
$$

Or,

$$
\begin{aligned}
\Rightarrow & a^{2}\left(\lambda^{3}+3 \lambda^{2}-\eta-3 \lambda \eta-3 \lambda^{2} \eta-\lambda^{3} \eta\right)+a\left(3 \lambda^{3}+9 \lambda^{2}-6 \eta \mu \lambda-3 \eta \mu \lambda^{2}-3 \eta \mu\right) \\
& +\left(2 \lambda^{3}+6 \lambda^{2}-2 \eta \mu^{2}-2 \eta \mu^{2} \lambda\right)=0
\end{aligned}
$$

Substituting the value from a equation (20) in the above equation and after simplifying the above equation, we have;

$$
\begin{aligned}
& \Rightarrow\left(\frac{\mu_{1}^{\prime} \delta(\lambda+1)-\lambda(\lambda+2)}{\lambda(\lambda+2)-\delta\left(\lambda^{2}+2 \lambda+1\right)}\right)^{2}\left(\lambda^{3}+3 \lambda^{2}-\eta-3 \lambda \eta-3 \lambda^{2} \eta-\lambda^{3} \eta\right) \\
& \quad+\left(\frac{\mu_{1}^{\prime} \delta(\lambda+1)-\lambda(\lambda+2)}{\lambda(\lambda+2)-\delta\left(\lambda^{2}+2 \lambda+1\right)}\right)\left(3 \lambda^{3}+9 \lambda^{2}-6 \eta \mu \lambda-3 \eta \mu \lambda^{2}-3 \eta \mu\right) \\
& \quad+\left(2 \lambda^{3}+6 \lambda^{2}-2 \eta \mu^{2}-2 \eta \mu^{2} \lambda\right)=0 \\
& \Rightarrow \lambda^{7}\left(2 \delta^{2}-\delta-\eta\right)+\lambda^{6}\left(14 \delta^{2}-3 \mu \delta^{2}-7 \delta-7 \eta+\mu \delta-\eta \mu \delta+3 \eta \mu\right) \\
& +\lambda^{5}\left(\mu^{2} \delta^{2}+\eta \mu^{2} \delta-6 \eta \mu \delta+6 \mu \delta-18 \mu \delta^{2}+18 \eta \mu-2 \eta \mu^{2}+36 \delta^{2}-19 \eta-7 \delta\right) \\
& +\lambda^{4}\left(5 \mu^{2} \delta^{2}+5 \eta \mu^{2} \delta-32 \eta \mu \delta+29 \mu \delta-36 \mu \delta^{2}+39 \eta \mu-10 \eta \mu^{2}+44 \delta^{2}-25 \eta-17 \delta\right) \\
& +\lambda^{3}\left(7 \mu^{2} \delta^{2}+4 \eta \mu^{2} \delta^{2}+5 \eta \mu^{2} \delta-16 \eta \mu \delta+6 \mu \delta-30 \mu \delta^{2}-16 \eta \mu^{2}+26 \delta^{2}-16 \eta-6 \delta\right) \\
& +\lambda^{2}\left(3 \mu^{2} \delta^{2}+7 \eta \mu^{2} \delta^{2}-9 \eta \mu \delta+6 \mu \delta-9 \mu \delta^{2}+12 \eta \mu-8 \eta \mu^{2}+6 \delta^{2}-4 \eta\right) \\
& +\lambda\left(2 \eta \mu^{2} \delta-2 \eta \mu \delta\right)
\end{aligned}
$$

The value of $\delta$ and $\eta$ can be calculated from the data directly by using the equation (19) and (21) respectively. After obtaining and substituting these values in equation (23) we get a seven-degree polynomial equation in $\lambda$, which is an unknown and analytical solution of $\lambda$ can be obtained. Once we get the value of $\lambda$ the value of other parameters a and $b$ can be easily obtained by using equations (20) and (16) respectively. After obtaining the estimates of the parameters the expected frequencies can be obtained by using the proposed probability model. 


\section{Application of the Model}

To illustrate the application of the model discussed above the data collected in NFHS-II (1998-99) ${ }^{(19)}$ and NFHS-III (2005$06)^{(20)}$ for the state Uttar Pradesh has been considered. For other major states data from NFHS-III (2005-06) has been used only. In this data information on all live births to a woman and their survival status at the time of survey has been collected. In case of death of child age at death for each child has been recorded separately. Women, who have no births in the preceding five years from the reference date of the survey, have been considered with the assumption that these females have already completed their family size. By this, we can avoid censored observations from the data. Childless females have been considered keeping the point in mind that all females should be taken for the experience of any child death.

The data set of the state Uttar Pradesh contains information on females. For NFHS-III data the whole state is divided into three categories namely Total, Urban and Rural according to their place of residence. The total number of women considered was 4673 and among them, the distribution of females according to their place of residence is 2106 (urban) \& 2567 (rural). For NFHS-II data the state of Uttar Pradesh contains information of 4385 females with 1003 urban and 3382 rural females. Also to check the suitability of the proposed model according to age groups the data of Uttar Pradesh is divided according to the present age group 30-39 and 40-49 years respectively and also according to a place of residence i.e. rural and urban.

\section{Results and Discussion}

The observed and expected number of frequencies of females according to the number of child death for the different domains of Uttar Pradesh i.e. Uttar Pradesh urban, rural, and as a whole are shown by Tables 1, 2 and 3 considering the data from NFHSIII and II. Tables also provide the estimated values of parameters of the model as derived in the above section. Table 3 reveals the suitability of the proposed model for the data set of Uttar Pradesh considered as a whole. It can be observed from the tables that the calculated value of chi-square is smaller than the corresponding tabular value at a 5 percent level of significance for each data set considered. By looking at the observed and expected frequencies one can conclude that the model graduates the data superbly. Thus, this refers to the suitability of the above-proposed model for the distribution of child death to the females in the domain for the state considered. The estimated value of the risk of child death to females is almost similar to the observed values reported in the NFHS-III report.

Tables 1 and 2 shows the observed and expected frequency of females experiencing child death with respect to the place of their residence (urban/rural) in Uttar Pradesh. It is observed from the tables that the estimate of the risk of child deaths in rural Uttar Pradesh is about 80 per thousand and 72 per thousand for NFHS-III and NFHS-II data respectively, which are much higher than the estimate of the risk of child deaths of urban females 50 per thousand and 55 per thousand for NFHS-III and NFHS-II data respectively. Table 3 suggests that the risk of child death is 63 per thousand and 69 per thousand for the females of Uttar Pradesh for NFHS-III and NFHS-II data respectively.

Table 1. Observed and Expected number of females according to the number of child deaths in urban females of Uttar Pradesh from NFHS-III \& II

\begin{tabular}{lllll}
\hline \multirow{2}{*}{$\begin{array}{l}\text { No. of Child } \\
\text { Deaths }\end{array}$} & NFHS-III & & NFHS-II & Expected Frequency \\
\cline { 2 - 5 } & Observed Frequency & Expected Frequency & Observed Frequency & 751.04 \\
1 & 1620 & 1620.89 & 750 & 154.57 \\
2 & 325 & 325.82 & 157 & 57.33 \\
3 & 107 & 104.25 & 60 & 23.51 \\
4 & 40 & 36.03 & 14 & 9.83 \\
$5+$ & 6 & 12.57 & 15 & 6.72 \\
Total & 8 & 6.44 & 7 & 1003 \\
\hline Parameters & 2106 & 2106 & 1003 & $\lambda=6.5481$ \\
& & $\lambda=5.9993$ & Parameters & $\widehat{a}=0.3622$ \\
& & $\widehat{a}=0.4274$ & & $b=6.2140$ \\
\hline Chi-Sq. (df) & & $4.324(2)$ & & $6.742(2)$ \\
p-value & & 0.115 & Chi-Sq. (df) & 0.034 \\
Risk of child death & & 0.0494 & p-value & 0.0551 \\
\hline
\end{tabular}


Table 2. Observed and Expected number of females according to the number of child deaths in rural females of Uttar Pradesh from NFHS-III \& II

\begin{tabular}{|c|c|c|c|c|}
\hline \multirow{2}{*}{$\begin{array}{l}\text { No. of Child } \\
\text { Deaths }\end{array}$} & \multicolumn{2}{|l|}{ NFHS-III } & \multicolumn{2}{|l|}{ NFHS-II } \\
\hline & Observed Frequency & Expected Frequency & Observed Frequency & Expected Frequency \\
\hline 0 & 1663 & 1649.70 & 2288 & 2248.99 \\
\hline 1 & 516 & 541.99 & 528 & 609.69 \\
\hline 2 & 227 & 220.34 & 301 & 269.25 \\
\hline 3 & 98 & 92.10 & 143 & 130.28 \\
\hline 4 & 43 & 38.07 & 71 & 64.32 \\
\hline 5 & 11 & 15.33 & 25 & 31.55 \\
\hline $6+$ & 9 & 9.47 & 14 & 15.18 \\
\hline $7+$ & & & 12 & 12.74 \\
\hline Total & 2567 & 2567 & 3382 & 3382 \\
\hline Parameters & & $\begin{array}{l}\widehat{\lambda}=6.8935 \\
\widehat{a}=0.6307 \\
\widehat{b}=7.2591\end{array}$ & Parameters & $\begin{array}{l}\widehat{\lambda}=7.8261 \\
\widehat{a}=0.4223 \\
\widehat{b}=5.4573\end{array}$ \\
\hline Chi-Sq. (df) & & $3.818(3)$ & Chi-Sq. (df) & $18.796(4)$ \\
\hline $\mathrm{p}$-value & & 0.282 & $\mathrm{p}$-value & 0.001 \\
\hline Risk of child death & & 0.0799 & Risk of child death & 0.0718 \\
\hline
\end{tabular}

Table 3. Observed and Expected number of females according to the number of child deaths in Uttar Pradesh from NFHS-III \& II

\begin{tabular}{lllll}
\hline \multirow{2}{*}{$\begin{array}{l}\text { No. of Child } \\
\text { Deaths }\end{array}$} & NFHS-III & \multicolumn{2}{l}{ NFHS-II } & Expected Frequency \\
\cline { 2 - 5 } 0 & 3283 & 3273.44 & 3038 & 3000.24 \\
1 & 841 & 864.47 & 685 & 762.90 \\
2 & 334 & 323.49 & 361 & 327.23 \\
3 & 138 & 128.42 & 157 & 154.47 \\
4 & 49 & 51.16 & 86 & 74.45 \\
5 & 15 & 20.04 & 30 & 35.63 \\
6 & 8 & 7.64 & 15 & 16.71 \\
$7+$ & 5 & 4.34 & 13 & 13.37 \\
Total & 4673 & 4673 & 4385 & 4385 \\
\hline Parameters & & $\lambda=6.7262$ & Parameders & $\lambda=7.5154$ \\
& & $\widehat{a}=0.5112$ & & $\widehat{a}=0.4010$ \\
& & $\widehat{b}=7.5836$ & & $b=5.4473$ \\
\hline Chi-Sq. (df) & & $3.167(3)$ & Chi-Sq. (df) & $14.883(4)$ \\
p-value & & 0.367 & p-value & 0.005 \\
Risk of child death & & 0.0632 & Risk of child death & 0.0686 \\
\hline
\end{tabular}

Tables 4 and 5 , shows the observed and expected frequencies of females experiencing child death with respect to their age in Uttar Pradesh i.e. female of current age 30-39 and 40-49 respectively for both the NFHS surveys. Here also the value of chisquare is insignificant at a 5 percent level of significance. From Tables 4 and 5 it is clear that the risk of child deaths is much higher for the females who belong to the age group 40-49 than the females who belong to the age group 30-39. For the age group 30-39, the risk of child death is 83 per thousand whereas 131 per thousand for the age group $40-49$ for the NFHS-III data. We get a similar pattern in the NFHS-II as well. For the age group 30-39 it is found to be 81 per thousand whereas 156 per thousand for the age group 40-49. This may be due to the fact that there is more chance of infant as well as child death if a female produces at higher ages. Although it can be seen that the difference between the two age groups 30-39 and 40-49 is decreased from NFHS-II to NFHS-III i.e. from 75 to 47 in the last 8 years. From Table 6 , we are getting the risk of child deaths for different states of India from NFHS-III which are highly contributing to the Infant and child mortality. 
Table 4. Observed and Expected number of females according to the number of child deaths by age group 30-39 in Uttar Pradesh from

\begin{tabular}{|c|c|c|c|c|}
\hline \multicolumn{5}{|c|}{ NFHS-III \& II } \\
\hline \multirow{2}{*}{$\begin{array}{l}\text { No. of Child } \\
\text { Deaths }\end{array}$} & \multicolumn{2}{|l|}{ NFHS-III } & \multicolumn{2}{|l|}{ NFHS-II } \\
\hline & Observed Frequency & Expected Frequency & Observed Frequency & Expected Frequency \\
\hline 0 & 1167 & 1159.31 & 969 & 962.46 \\
\hline 1 & 325 & 340.74 & 287 & 305.61 \\
\hline 2 & 120 & 113.95 & 130 & 113.94 \\
\hline 3 & 39 & 37.75 & 41 & 42.85 \\
\hline \multirow{2}{*}{$4+$} & 18 & 17.25 & 14 & 15.74 \\
\hline & & & 8 & 8.40 \\
\hline Total & 1669 & 1669 & 1449 & 1449 \\
\hline \multirow[t]{3}{*}{ Parameters } & & $\lambda=4.4360$ & Parameters & $\widehat{\lambda}=5.5399$ \\
\hline & & $\widehat{a}=0.6953$ & & $\widehat{a}=0.6770$ \\
\hline & & $\widehat{b}=7.6264$ & & $\widehat{b}=7.6436$ \\
\hline Chi-Sq. (df) & & $1.173(1)$ & Chi-Sq. (df) & $3.733(2)$ \\
\hline $\mathrm{p}$-value & & 0.279 & p-value & 0.155 \\
\hline Risk of child death & & 0.0836 & Risk of child death & 0.0814 \\
\hline
\end{tabular}

Table 5. Observed and Expected number of females according to the number of child deaths by age group 40-49 in Uttar Pradesh from NFHS-III \& II

\begin{tabular}{|c|c|c|c|c|}
\hline \multirow{2}{*}{$\begin{array}{l}\text { No. of Child } \\
\text { Deaths }\end{array}$} & \multicolumn{2}{|l|}{ NFHS-III } & \multicolumn{2}{|l|}{ NFHS-II } \\
\hline & Observed Frequency & Expected Frequency & Observed Frequency & Expected Frequency \\
\hline 0 & 918 & 925.52 & 801 & 784.89 \\
\hline 1 & 414 & 403.97 & 353 & 382.80 \\
\hline 2 & 189 & 187.58 & 217 & 209.50 \\
\hline 3 & 86 & 84.95 & 115 & 114.23 \\
\hline 4 & 33 & 36.76 & 72 & 60.28 \\
\hline 5 & 12 & 15.09 & 26 & 30.41 \\
\hline $6+$ & 11 & 9.13 & 12 & 14.58 \\
\hline $7+$ & & & 12 & 11.31 \\
\hline Total & 1663 & 1663 & 1608 & 1608 \\
\hline Parameters & & $\begin{array}{l}\widehat{\lambda}=5.0210 \\
\widehat{a}=0.7932 \\
\widehat{b}=5.2417\end{array}$ & Parameters & $\begin{array}{l}\widehat{\lambda}=5.8032 \\
\widehat{a}=0.7385 \\
\widehat{b}=3.9807\end{array}$ \\
\hline Chi-Sq. (df) & & $1.734(3)$ & Chi-Sq. (df) & $6.341(4)$ \\
\hline $\mathrm{p}$-value & & 0.629 & $\mathrm{p}$-value & 0.175 \\
\hline Risk of child death & & 0.1314 & Risk of child death & 0.1565 \\
\hline
\end{tabular}

Table 6. Estimated values of the parameters for different Indian states by NFHS-III

\begin{tabular}{|c|c|c|c|c|c|c|}
\hline \multirow{2}{*}{ States } & \multicolumn{3}{|c|}{ Parameters } & \multirow{2}{*}{ chi-square } & \multirow{2}{*}{$\begin{array}{l}\text { Degree of } \\
\text { freedom }\end{array}$} & \multirow{2}{*}{ Risk of child death } \\
\hline & $\widehat{\lambda}$ & $\widehat{a}$ & $\widehat{b}$ & & & \\
\hline \multicolumn{7}{|l|}{ Northern States } \\
\hline Uttar Pradesh & 6.7262 & 0.5112 & 7.5836 & 3.2568 & 4 & 0.0632 \\
\hline Bihar & 6.3683 & 0.4298 & 7.3630 & 3.3813 & 4 & 0.0552 \\
\hline Madhya Pradesh & 4.5315 & 0.3213 & 3.1414 & 0.1659 & 2 & 0.0928 \\
\hline Rajasthan & 5.6221 & 0.3317 & 5.2801 & 5.0062 & 4 & 0.0591 \\
\hline \multicolumn{7}{|l|}{ Eastern States } \\
\hline Orissa & 4.0157 & 0.4699 & 7.7448 & 1.1698 & 1 & 0.0572 \\
\hline West Bengal & 4.5174 & 0.2537 & 6.7115 & 1.3115 & 1 & 0.0364 \\
\hline \multicolumn{7}{|l|}{ Western States } \\
\hline Gujarat & 4.0025 & 0.3172 & 6.9107 & 0.3739 & 1 & 0.0439 \\
\hline
\end{tabular}




\begin{tabular}{lllllll}
\hline \multicolumn{1}{c}{ Table 6 continued } & \multicolumn{7}{c}{} \\
\hline Maharashtra & 4.7816 & 0.2372 & 8.5353 & 7.2130 & 1 & 0.0270 \\
\hline Southern States & & & & & 4 & 0.0333 \\
\hline Andhra Pradesh & 4.0157 & 0.3080 & 8.9412 & 2.0712 & 1 & 0.0340 \\
Karnataka & 4.2088 & 0.3253 & 9.2339 & 2.6655 & - & 0.0165 \\
Kerala & 1.9095 & 0.1497 & 8.9482 & 0.4122 & - & 0.0400 \\
Tamil Nadu & 2.9724 & 0.3282 & 7.8670 & 3.2133 & & \\
\hline
\end{tabular}

From the results discussed above, we may conclude that the model proposed here may be considered to be suitable to describe the distribution of a number of child death to females. The remarkable utility of this model is that it provides a clear visualization about the unobservable risk of child deaths and also provides the average children ever born to female through the distribution of females according to the number of child deaths. Thus, this model provides a new dimension for comparing the risk of child death to females in different regions of Uttar Pradesh and other states.

\section{Conclusion}

In this study, we suggest a 'model for child death' which we believe will describe the pattern of mortality adequately for a wide variety of experiences. Although the model may not always give a fit close enough for certain purposes it reproduces the risk of child death and the mean number of children ever born. The model is appropriate and applicable to other similar data. The mathematical model gives an adequate representation of child deaths among all females of Uttar Pradesh and preliminary studies give different experiences indicates its wide applicability. The model can be used to calculate the average number of children ever born through the distribution of a number of child death. One may modify the proposed model by taking some truncated probability distribution for the number of children ever born to the females, which would be done separately in the future.

\section{References}

1) Bhuyan KC. Fertility differential according socio-economic status and family planning adoption in rural Bangladesh. Sankhya: Indian Journal of Statistics, Series B. 1996;58(2):302-322. Available from: https://www.jstor.org/stable/25052954?seq=1.

2) Brijesh PS, Shweta D, Sandeep S. Does infant mortality regulate fertility behaviour ofwomen in Uttar Pradesh? A causality test analysis. Demography India. 2017;46(1):38-47. Available from: http://demographyindia.in/article_document/27/265443.pdf.

3) Gompertz B. On the nature of the function expressive of the law of human mortality, and on a new mode of determining the value of life contingencies. Proceedings of the Royal Society of London. 1825;2(0):253-253. Available from: https://doi.org/10.1098/rspl.1815.0271.

4) Keyfitz N. Introduction to the Mathematics of Population. Massachusetts. Addison-Wesley. 1968.

5) Heligman L, Pollard JH. The age pattern of mortality. Journal of the Institute of Actuaries. 1980;107(1):49-80. Available from: https://dx.doi.org/10.1017/ s0020268100040257.

6) Hartman M. Infant and Child Mortality. 1982.

7) Cheo MK. Fitting of age pattern of infant and child mortality with the weibull survival distribution. Asian and Pacific Census Forum. 1981;7(4):10-13. Available from: https://pubmed.ncbi.nlm.nih.gov/12337611/.

8) Krishnan P, Jin Y. A statistical model of infant mortality. Janasamkhya. 1993;11(2):67-71. Available from: https://pubmed.ncbi.nlm.nih.gov/12292837/.

9) Chauhan RK. Graduation of infant deaths by age. Demography India. 1997;26(2):261-274.

10) Brijesh PS, Tapan KR, Sonam M, Gunjan S. Deaths by age in Madhya Pradesh and its estimation in mortality. Health and Development. Ranjan A, Foundation M, editors;Shyam Institute, Bhopal. 2014.

11) Brijesh PS, Dixit S, Roy TK. Stochastic Analysis of Infant Deaths by Age and Estimation of Parameters. Journal of Institute of Science and Technology. 2015;20(1):1-5. Available from: https://dx.doi.org/10.3126/jist.v20i1.13903.

12) Bhuyan KC, Deogratias R. On a probability model for child mortality pattern in north-eastern Libya. Turkish Journal of Population Studies. 1999;21:33-37.

13) Alok K, Singh BP, Srivastava S, Kns Y. Level and Parent of Infant and Child Mortality in India Subcontinent. Journal of Empirical Research in Social Science. 2005;1(1):39-57.

14) Brijesh PS, Neha S, Tapan KR, Gunjan S. On the pattern of child loss in Madhya Pradesh. Mortality, Health and Development. Ranjan A, Foundation M, editors;Shyam Institute, Bhopal. 2012.

15) Singh KK, Brijesh PS, Neha S. A probability model for number of child death for fixed parity. Demography India. 2011;40(2):55-68.

16) Singh KK, Brijesh PS, Neha S. A probabilistic study of variation in number of child deaths. Journal of Rajasthan Statistical Association. $2012 ; 1(1): 54-67$.

17) Brijesh PS, Shweta D, Tapan KR. On some compounded poisson distributions with applications to the pattern of number of child deaths. Journal of Probability and Statistical Sciences. 2016;14(2):203-209.

18) Brijesh PS, Das UD, Gunjan S. Development of a discrete probability distribution and its application to the pattern of child deaths. Accepted in Journal of Scientific Research. 2021;65(5).

19) National Family Health Survey-II (NFHS-II). International Institute of Population Sciences, Mumbai . 1998. Available from: https://www.dhsprogram. com/pubs/pdf/FRIND2/FRIND2.pdf.

20) National Family Health Survey-III (NFHS-III). International Institute of Population Sciences, Mumbai. 2005-06. 2005. Available from: http://rchiips.org/ nfhs/nfhs3.shtml. 